\title{
Tears of the fascia cruris demonstrate characteristic sonographic features: a case series analysis
}

\author{
Sarah Morton 1 \\ Otto Chan ${ }^{2}$ \\ Nick Webborn ${ }^{3}$ \\ Melanie Pritchard² \\ Dylan Morrissey ${ }^{4}$
}

1 Centre for Sports and Exercise Medicine, Queen Mary University of London, UK

2 BMI London Independent Hospital, London, UK

3 Centre for Sports and Exercise Science and Medicine, University of Brighton, Eastbourne, UK

4 Centre for Sports and Exercise Medicine William Harvey Research Institute Queen Mary University of London, UK; BMI London Independent Hospital, London, UK

Corresponding author:

Dylan Morrisey

Centre for Sports and Exercise Medicine, Queen Mary University of London

Mile End Hospital, Bancroft road, E1 4DG, London, UK

E-mail: d.morrissey@qmul.ac.uk

\section{Summary}

Background: fascia cruris (FC) tears have recently been recognised in the literature, although little is known about their characteristic ultrasound findings. The aim was to describe the echo-graphic features of FC tears in order to improve recognition and diagnosis.

Methods: the ultrasound reports and images of $>600$ patients attending a specialist musculoskeletal clinic for Achilles tendon ultrasound scans between October 2010-May 2014 were reviewed. Any patient diagnosed with a FC tear had a structured data set extracted. All ultrasound images were performed by one consultant radiologist. Bilateral Achilles images were available for analysis.

Results: sixteen patients from $>600$ subjects were diagnosed with a FC tear. Fourteen subjects were male and two female (mean age 37.8 ; range 2361 ), with seven elite level sports men. Nine tears were right sided and seven left, with eight situated laterally and seven medially. Seven of the tears were situated in the musculotendinous junction.
Symptomatic Achilles tendinopathy co-existed in ten of sixteen subjects (average transverse diameter of Achilles tendon = 7.1 $\pm 2.0 \mathrm{~mm}$ ).

Conclusion: FC tears should be considered in the differential diagnoses for Achillodynia, diagnosed using their characteristic ultrasound findings, with a hypoechoic area at the medial or lateral attachment to the Achilles tendon in the transverse plane.

KEY WORDS: Achilles, fascia cruris, MRI, tear, ultrasound.

\section{Introduction}

The Achilles tendon is the largest tendon in the body consisting of fibres from both gastrocnemius and soleus, and is commonly injured, including Achilles tendinopathy, partial tears and Achilles rupture ${ }^{1,2}$. The fascia cruris is connective tissue that splits the leg into its three recognised muscular compartments: the anterior, posterior and lateral compartments ${ }^{3}$. Within the posterior compartment Stecco et al. (2013) have shown that the fascia cruris divides around the Achilles tendon to form the paratenon, which is then implicated in the production of pain in tendinopathy, due to its high vascularity and innervation ${ }^{4}$. This is in contrast to work by Carmont et al. (2011) who distinguished these as separate layers on dissection in some subjects ${ }^{5}$. It has also been shown that the fascia cruris is thickened in people with tendinopathy, with a mean of $1.30 \mathrm{~mm}$ versus $1.11 \mathrm{~mm}$ in a normal subject ${ }^{4}$. The paratenon remains partially separated from the Achilles tendon by loose connective tissue 4 . Ultrasound (US) and magnetic resonance imaging (MRI) are recognised as useful imaging techniques, when the clinical history and examination does not immediately distinguish the cause of Achillodynia ${ }^{2}$. $\mathrm{MRI}$ can be used to distinguish the fascia cruris and paratenon, although the paratenon can become difficult to distinguish near the calcaneal insertion point ${ }^{4,6}$. MRI can be used to measure the thickness of the fascia cruris and to detect tendinopathic changes within the Achilles tendon 4,6 . Ultrasound has the advantage over MRI in that it can provide dynamic assessments of the tendon, has better soft tissue resolution and can establish the grade of neovascularisation present, particularly important in tendinopathic subjects ${ }^{2,6}$.

Until recently injuries to the fascia cruris had not been recognised as a cause of Achillodynia; indeed there is 
only one paper present in the literature describing a case series of nine athletes with tears of the fascia cruris from the attachment to the paratenon and Achilles tendon ${ }^{7}$. The aim of our study was to enable musculoskeletal clinicians and radiologists to differentially diagnose fascia cruris tears, by analysing and describing a consecutive radiological case series of diagnosed patients diagnosed with a fascia cruris tear from a large long-term cohort of patients with Achillodynia.

\section{Materials and methods}

The ultrasound reports and images of all patients who attended for Achilles tendon ultrasound scans between October 2010 and May 2014 were reviewed retrospectively. Patients were referred mainly for Achillodynia from a large referral base including sports medicine clinics, team doctors and physiotherapists. Any patient diagnosed in their ultrasound report as having a fascia cruris tear was identified in our database and the data extracted, including age, gender, level of sport participated in, size and positioning of tear. Any additional imaging techniques used for these subjects, such as MRI, were also obtained and reviewed. Ethical approval for this study was obtained from Queen Mary, University of London Ethics of research Commitee. All work was carried out as per the standards described by Padulo et al. (2013) ${ }^{8}$.

\section{Ultrasound Imaging}

The ultrasound images reviewed were all performed by the same experienced musculoskeletal consultant radiologist with over 30 years' experience. The same ultrasound scanner was used throughout the study (Elegra, Siemens, Erlangen Germany). Patients attending for Achilles tendon scanning at the London Independent Hospital are always scanned by $\mathrm{OC}$ in the same position to allow for comparison at all-time points. The patients are placed in a long sitting position, with their hips flexed and externally rotated, their knees at $90^{\circ}$ and their ankles in a neutral position i.e. a seated frogs leg position ${ }^{9}$. A $13 \mathrm{MHz}$ ultrasound probe was used. A fascia cruris tear was identified if areas of hypoechoic changes at the medial or lateral attachment to the Achilles tendon in the transverse plane were seen, while the paratenon on the dorsal surface of the tendon was normal. A fascia cruris tear was most commonly viewed in transverse section and to enable the optimum view of the fascia the probe was titled, as per the European Society of Musculoskeletal Radiology technical guidelines for examination of the ankle ${ }^{9}$. In the patients scanned, longitudinal and transverse static and dynamic video images were obtained for both Achilles to allow comparison between sides, alongside Power Doppler imaging. Data regarding maximal antero-posterior tendon diameter, shown by Fredberg et al. (2008) to be a reliable measure, and neovascularisation grading based on a modified Ohberg scale were recorded at the time of examination ${ }^{10-12}$. It was also noted on the report at the time of scanning if pain was precipitated by the pressure of the ultrasound probe (sonopalpation) at the area of change detected on ultrasound. Subjects were asked if they had Achilles tendinopathy symptoms alongside the pain from the tear, and data regarding this recorded. A multi-disciplinary team approach is used at the time of ultrasound scanning at this specialist musculoskeletal centre with at least one sports physician and consultant physiotherapist present alongside the consultant radiologist to allow discussions and confirmation of diagnoses. From this, the data could then be extracted and direct comparisons made when reviewing images and reports.

\section{Data analysis}

All data extracted was coded to ensure confidentiality and anonymity in Microsoft Excel. SPSS version 20 was used for analysis of descriptive statistics. Images were also described qualitatively.

\section{Results}

Sixteen patients diagnosed with a fascia cruris tear between October 2010 and May 2014 were extracted from a data set of over 600 subjects. There were fourteen males and two females (mean age 37.8 years, range 23-61), with seven elite level sportsmen. The patient characteristics can be seen in Table 1.

\section{Ultrasound Findings}

Table 2 shows the characteristics of the tears in the sixteen patients. There were nine right fascia cruris tears and seven left fascia cruris tears. Eight were situated laterally to the tendon and seven medial. Seven of the tears were in the musculotendinous junction, of which five were lateral and two medial. One patient (with a asterisk in Tab. 2 below) was found to have both a large medial fascia cruris tear and a lateral intratendinous tear, as described by Morton et al. (2013) ${ }^{13}$. The mean transverse diameter of the Achilles tendon was $7.1 \pm 2.0 \mathrm{~mm}$. One of the tendon diameters fell close to the normal control value of $4.4 \mathrm{~mm}$, as reported by Leung and Griffth (2008), with a tendon diameter of $4.6 \mathrm{~mm}^{14}$. All of the other tendon diameters were greater than $5.6 \mathrm{~mm}$, classified as tendinopathic by Leung and Griffth, with only two of the tendons (including the "normal" $4.6 \mathrm{~mm}$ tendon) having a neovascularisation grade of $0^{11,14}$. None of the sixteen patients were found to have more than one fascia cruris tear.

The images below show the fascia cruris tear on ultrasound (Figs. 1-5) and MRI (Figs. 6, 7).

Table 1. Subjects characteristics.

\begin{tabular}{ll}
\hline Feature & Fascia crural tear group \\
\hline Sex (male: female) & $14: 2$ \\
$\begin{array}{l}\text { Mean age in years (range) } \\
\text { Level of sport (elite: amateur: }\end{array}$ & $37.8(23-61)$ \\
unavailable) & $7: 3[6]$ \\
\hline
\end{tabular}


Table 2. Characteristics of the fascia cruris tears observed on ultrasound based on the Del Buono et al. classification ${ }^{6}$.

\begin{tabular}{|c|c|c|c|c|c|}
\hline Side & $\begin{array}{l}\text { Med-lat } \\
\text { Position }\end{array}$ & $\begin{array}{l}\text { Ant-sup } \\
\text { position }\end{array}$ & $\begin{array}{l}\text { Transverse diameter } \\
\text { of Achilles tendon }(\mathrm{mm})\end{array}$ & $\begin{array}{l}\text { Neo-vasc } \\
\text { Grade }\end{array}$ & $\begin{array}{l}\text { Symptomatic } \\
\text { Tendinopathy? }\end{array}$ \\
\hline Left & Lateral & Proximal Free Achilles & 5.2 & 3 & Yes \\
\hline Left & Lateral & MTJ & 6.5 & 1 & Yes \\
\hline Right & Medial & Mid portion free Achilles & 12.5 & 3 & Yes \\
\hline Right & Medial & MTJ & 6.8 & 3 & Yes \\
\hline Right & Lateral & Distal free Achilles & 4.6 & 0 & No \\
\hline Left & - & - & 8.0 & 1 & Yes \\
\hline Right* & Medial & - & 7.5 & 4 & Yes \\
\hline Right & Lateral & Mid portion free Achilles & 6.1 & 0 & No \\
\hline Right & Lateral & MTJ & - & - & - \\
\hline Right & Lateral & MTJ & - & - & No \\
\hline Right & Medial & - & - & - & - \\
\hline Left & Lateral & MTJ & - & - & Yes \\
\hline Left & Lateral & MTJ & 6.6 & 1 & No \\
\hline Right & Medial & Proximal free Achilles & 6.0 & 3 & Yes \\
\hline Left & Medial & Proximal free Achilles & 8.8 & 2 & Yes \\
\hline Left & Medial & MTJ & 6.7 & 2 & Yes \\
\hline
\end{tabular}

MTJ = musculotendinous junction; - = data unavailable; med = medial; lat = lateral; ant = anterior; sup = superior; neo-vasc $=$ neovascularisation; ${ }^{*}$ = fascia cruris tear and lateral intratendinous tear.

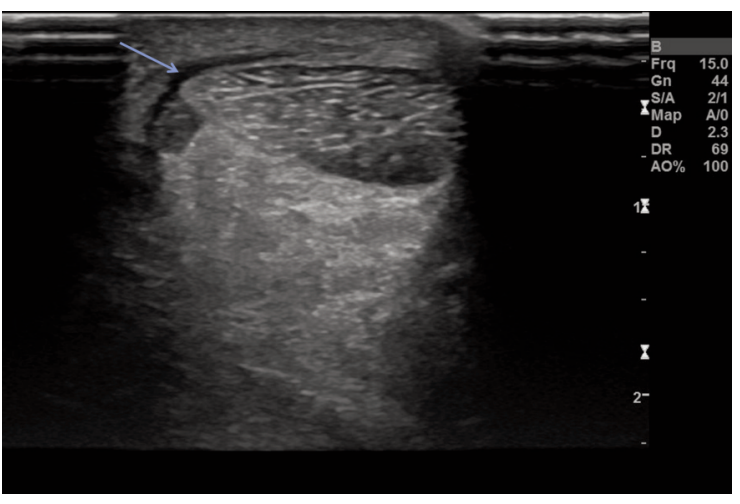

Figure 1. Ultrasound scan of a left sided fascia cruris tear shown in transverse section with patient seated in a "frog's leg" position and the probe tilted (see methods for full description).

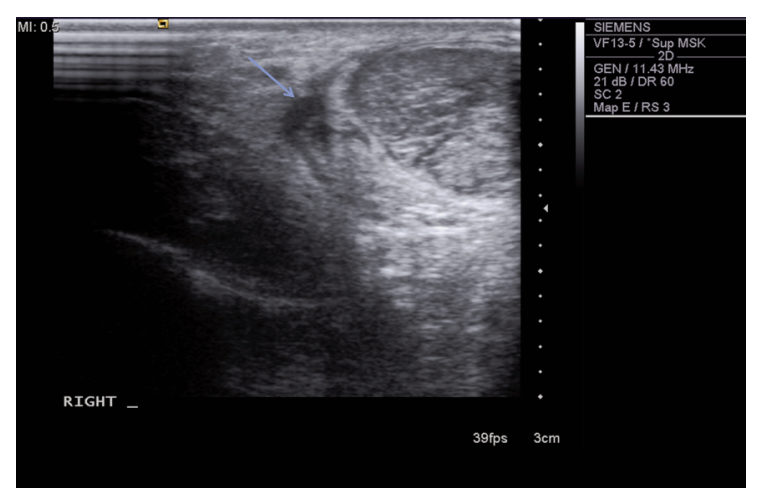

Figure 2. Ultrasound scan of a right sided fascia cruris tear shown in transverse section with patient seated in a "frog's leg" position and the probe tilted.

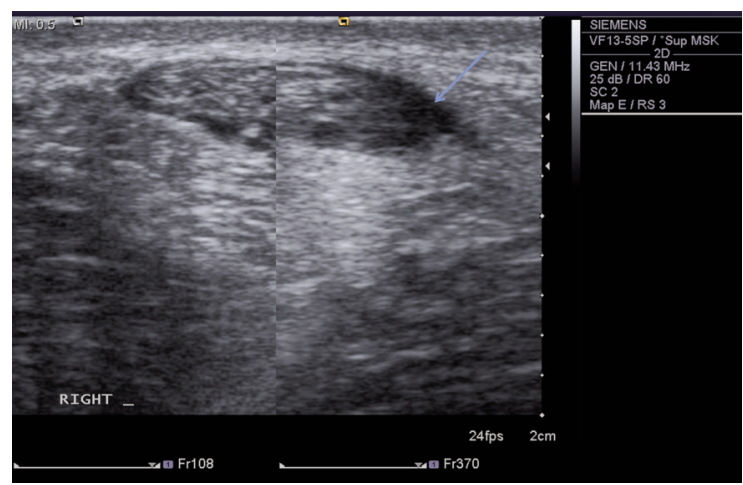

Figure 3. Ultrasound with comparison of a normal right sided tendon (as marked on scan) to a fascia cruris tear on the left (blue arrow) shown in transverse section with patient seated in a "frog's leg" position and the probe tilted.

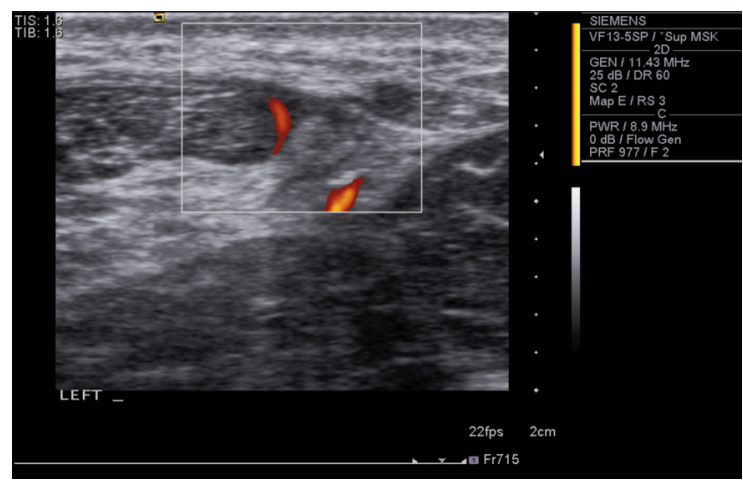

Figure 4. Neovascularisation on Power Doppler of the left sided fascia cruris tear (same as shown in Figure 3) shown in transverse section with patient seated in a "frog's leg" position and the probe tilted. 


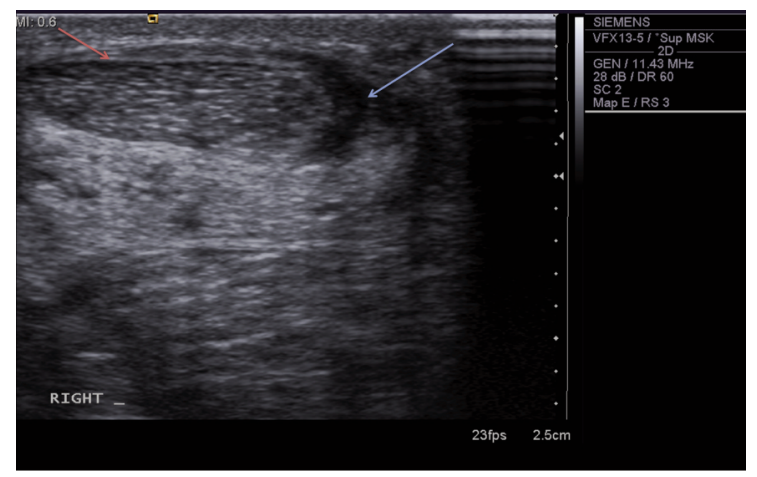

Figure 5. Ultrasound image showing right sided fascia cruris tear (blue arrow) with the top edge of a separate intratendinous tear being just visible (red arrow) shown in transverse section with patient seated in a "frog's leg" position and the probe tilted. The intratendinous tear is shown as an echopoor area within the tendon but not through the tendon periphery, whereas the fascia cruris tear is a hypoechoic area outside the tendon.

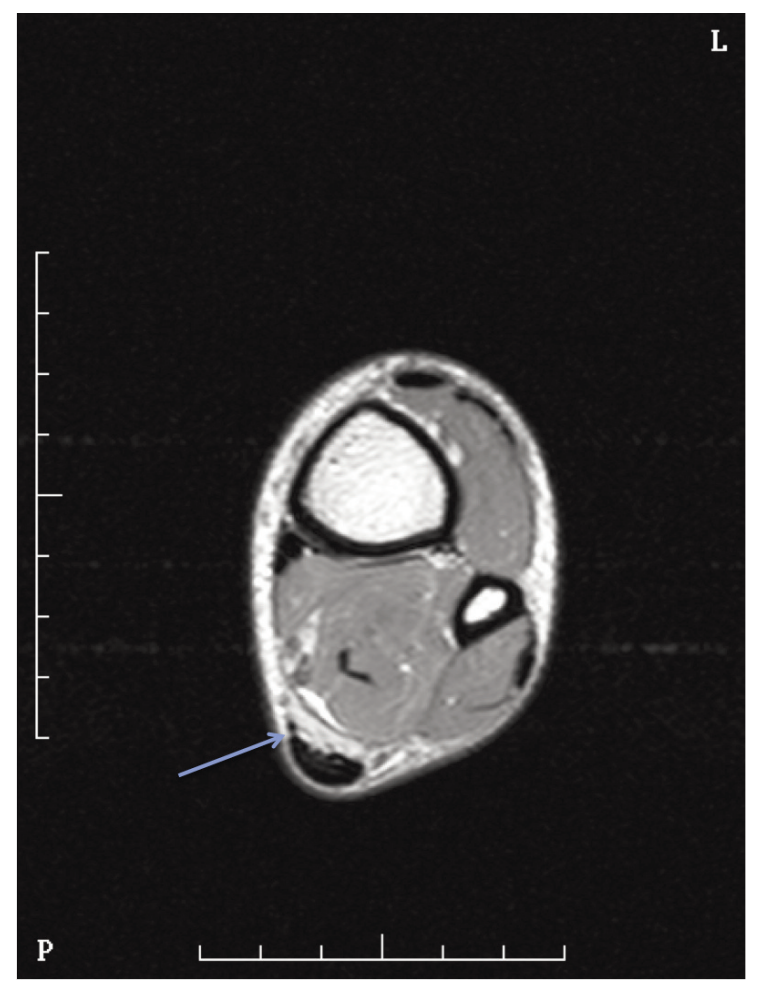

Figure 6. MRI image of fascia cruris in transverse section (blue arrow) with signal enhancement on the medial side of the Achilles tendon.

\section{Clinical Findings}

Of the sixteen subjects, only two reported being asymptomatic for tendinopathy. The remaining ten subjects (two data sets unknown) reported also having concurrent symptoms consistent with Achilles tendinopathy, in addition to the pain from the fascia cruris tear. From the reports it was noted that the

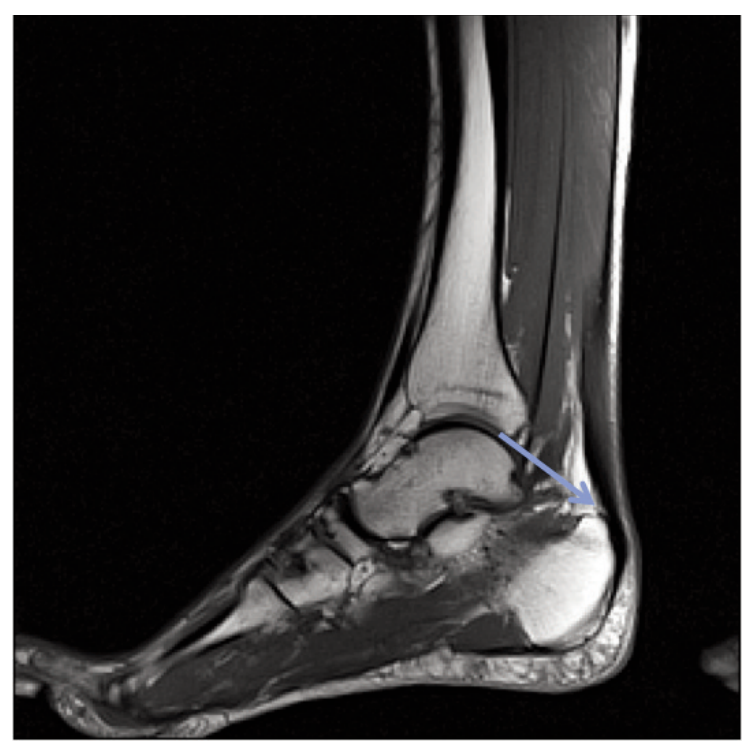

Figure 7. MRI image of fascia cruris in sagittal section (blue arrow) with signal enhancement seen just superior to the calcaneus.

subjects often reported a new pin-point tenderness without morning stiffness of an acute onset, unlike tendinopathy where patients typically present with a dull ache of gradual onset throughout their tendon and morning stiffness. Subjects also reported the ability of being able to jog but not push off or spring due to the pin-point pain. On clinical examination there was an area of maximal tenderness, consistent with the area the subject described and also consistent with the changes observed on ultrasound.

\section{Discussion}

This study reports sixteen fascia cruris tears (9 right; 7 left) presenting to a specialist MSK radiologist over the course of four years. Fourteen of the tears were present in men and only two in women. Seven out of ten (six data sets unavailable) were elite level athletes, including five current professional footballers. The mean age of presentation was 37.8 years with a range of 23 to 61 years. Ten of the sixteen subjects had concurrent symptomatic Achilles tendinopathy, with a mean tendon diameter of $7.1 \pm 2.0 \mathrm{~mm}$. These findings should be considered as a possible differential diagnosis when performing ultrasound scans of patients with Achillodynia.

\section{Strengths and weaknesses of study}

This study builds on the study by Webborn et al. (2014) by showing an additional sixteen cases with greater detail regarding the ultrasound findings, in what is likely to be a rare condition ${ }^{7}$. It looks in detail at the imaging findings and to establish other co-presentations of pathologies to aid clinicians in diagnosis. 
However, this study is a retrospective study based on ultrasound reports and images. Ideally confirmation of the ultrasound findings at surgery would be required, although it is recognised that elite athletes will want to aim to avoid surgery and invasive procedures. Therefore it is likely that the images and videos, alongside the ultrasound reports, are sufficient to establish this diagnosis as a cause of Achillodynia. Further work is required that includes long-term follow-up of these patients, especially in regards to changes seen on ultrasound and the optimum treatment regime for this injury.

\section{Comparison to literature}

As described above, this study adds numbers to this newly recognised diagnosis ${ }^{7}$. These findings do, however, differ somewhat from other studies on fascia cruris tears ${ }^{7}$. Previous work suggested a lower mean age of presentation (34.8 years) with a range that was skewed towards a younger population (11-48 years), although it is recognised that the numbers in both studies are relatively small. It should also be noted that no subject during the four year period, described in this study, was found to have more than one fascia cruris tear, unlike two patients in the Webborn et al. (2014) study who each experienced separate bilateral fascia cruris tears ${ }^{7}$. One subject in this study was found to have a medial fascia cruris tear and a separate posterior intratendinous tear within the same tendon (Fig. 5). Intratendinous tears have recently been described in the literature as echo poor areas detected on US situated within the tendon associated with a clinical history of point tenderness ${ }^{13}$. This therefore differs from the fascia cruris tear which is separate from the Achilles tendon ${ }^{4}$. The finding of co-existing pathologies is important as it indicates that clinicians must carefully elicit the correct history to endeavour to guide diagnosis; whilst in this case both were found to be painful it may be that two pathologies can co-exist, of which only one is currently the cause of Achillodynia.

Table 2 shows that there were nine right fascia cruris tears and seven left fascia cruris tears. Eight were situated lateral to the tendon and seven medial. Seven of the tears were found at the musculotendinous junction, of which five were lateral and two medial. This therefore makes it difficult to come to any conclusion as to whether it is the fascia cruris from the gastrocnemius or soleus that is more likely injured ${ }^{1}$. In the Webborn et al. (2014) study seven of the eleven tears described were lateral and it was suggested that this was due to tension through the fascia as the foot pronates and supinates ${ }^{7}$. However, due to the essentially equal numbers seen on each side in our data any such mechanism would need to be driven both by supination for lateral tears and pronation for medial.

A comparison study between controls and cases in 2007 showed a statistically significant difference in antero-posterior diameter of the Achilles tendon (5.6 $\mathrm{mm}$ case $v 4.4 \mathrm{~mm}$ control $)^{14}$. Based on these values, only one of the tendon diameters described in Table 2 fell close to this normal control value (4.6 $\mathrm{mm}$ in the study) with all the others greater than 5.6 $\mathrm{mm}$. This would therefore be consistent with Achilles tendinopathy being present alongside the fascia cruris tear. This also correlates with the degree of neovascularisation present, with only two (including the 'normal' $4.6 \mathrm{~mm}$ tendon) having a neovascularisation grading of 0 . It should be noted that despite the tendon appearing tendinopathic on ultrasound with an increased tendon diameter, two subjects reported being asymptomatic for tendinopathy; the remainder presented with symptoms consistent with tendinopathy in addition to the pain from the fascia cruris tear, again strengthening the need to carefully elicit the history and to always consider co-existing pathologies. In the Webborn et al. (2014) study only two of the nine patients had ultrasound changes consistent with Achilles tendinopathy ${ }^{7}$. It could be the case, as Frankyln-Miller et al. (2009) suggested, that fascial pathology precedes tendinopathy, and thereby fascia cruris tears proceed tendinopathy, although the timelines for the subjects described in this study do not correspond to this theory completely ${ }^{15}$. It may therefore be that previous Achilles tendinopathy predisposes a patient to a fascia cruris tear, or vice versa, but the important clinical point is that fascial tears can occur with or without tendinopathic changes.

Anecdotally, from the ultrasound reports, the subjects all presented with similar symptoms. The subjects often reported pin-point tenderness without morning stiffness of an acute onset, unlike tendinopathy where patients typically present with a dull ache of gradual onset throughout their tendon and morning stiffness. Subjects also reported the ability of being able to jog but not push off or sprint due to the pin-point pain. Again anecdotally on clinical examination there was an area of maximal tenderness, consistent with the area the subject described and also consistent with the changes observed on ultrasound. These descriptions are consistent with the description by Webborn et al., ${ }^{7}$ and should be actively described in future prospective studies. It should be noted that this study only describes the diagnosis of a FC tear and as a result the treatment used in the specialist MSK centre is not described; further work on this is required to establish its effectiveness.

Figures 1-5 show echo-poor areas seen on ultrasound that correspond to the area of pin-point pain described by the subjects. Figure 3 shows the comparison of a normal to an abnormal image, with Figure 4 showing the neovascularisation that has been found to grow into these tears, a useful sign on ultrasound if unsure of the diagnosis. The MRI images (Figs. 6, 7) also show the fascia cruris tear but is perhaps more difficult to detect and it may therefore be that ultrasound imaging is required if a fascia cruris tear is suspected on MRI. It should also be noted that ultrasound imaging is less costly than an MRI, allows side-to-side comparison, allows dynamic movemen- 
tand can often be organised in a more timely manner, for example immediately in a clinic setting. However, unlike MRI, ultrasound does partially rely on operator skill with subtle probe manipulation required to optimally image the fascia cruris. This level of skill needs to be considered by physicians managing complex cases and if necessary an expert radiological opinion sought.

The main differential diagnosis for a fascia cruris tear is peritendinitis or an intratendinous tear ${ }^{13}$. However US features differ in that peritendinitis is said to cause altered intratendinous structure and poorly defined Achilles tendon borders ${ }^{16}$. As can be seen clearly in Figure 1 and 2 above in a fascia cruris tear the Achilles tendon itself is not affected and it is instead outside the tendon that the echopoor area is seen. An intratendinous tear is an echpoor area situated centrally and extending to, but not through, the tendon periphery and so again differs from the images seen above ${ }^{13}$.

\section{Implications for Clinicians}

Fascia cruris tears should be considered in the differential diagnoses for Achilles pathology. Such tears can be diagnosed on ultrasound but the examiner needs to be mindful of transversely tilting the probe at the tendon margins to optimally evaluate the fascia cruris, alongside a consistent clinical history and examination. Other imaging techniques such as MRI may also be useful to corroborate ultrasound findings. Fascia cruris tears appear to be more common in patients with tendinopathy but tendinopathy does not have to be present for a tear to occur, while tears also appear to be more common in subjects performing at a high sporting level. Tears need to be differentiated from acute paratendinitis where the clinical picture and sonographic findings are different. Co-existing pathologies should be considered and the exact cause of pain elicited. Prospective research that includes the sensitivity and specificity of both imaging and examination findings are required alongside assessment of optimal treatments, and its prevalence outside of a specialist centre.

\section{Conclusion}

FC tears are a newly recognised differential for Achillodynia. This study shows they can be diagnosed using their characteristic ultrasound findings of a hypoechoic area at the medial or lateral attachment to the Achilles tendon in the transverse plane. The diagnosis should be supported with a consistent clinical history and examination. Concurrent pathologies should be considered alongside the presence of a FC tear.

\section{Acknowledgements}

Dr. Morrisey is part funded by the NIHR/HEE senior clinical lecturer scheme. This article presents independent research part-funded by the National Institute for Health Research (NIHR). The views expressed are those of the Authors and not necessarily those of the NHS, the NIHR or the Department of Health.

\section{References}

1. Doral MN, Alam M, Bozkurt M, Turhan E, Atay OA, Donmez G, Maffulli N. Functional anatomy of the Achilles tendon. Knee Surg Sports Traumatol Arthrosc. 2010;18:638-643.

2. Asplund CA, Best TM. Achilles tendon disorders. BMJ. 2013;346:f1262.

3. Bradshaw C, Hislop M, Hutchinson M. Shin Pain. In: Brukner P, Khan K. eds Clinical Sports Medicine. Australia, McGrawHill. 2010;30:555.

4. Stecco C, Cappellari A, Macchi V, Porzionato A, Morra A, Berizzi A, De Caro R. The paratendineous tissues: an anatomical study of their role in the pathogenesis of tendinopathy. Surg Radiol Anat. 2014;36:561-572.

5. Carmont MR, Highland AM, Rochester JR, Paling EM, Davies MB. An anatomical and radiological study of the fascia cruris and paratenon of the Achilles tendon. Foot Ankle Surg. 2011; 17:186-192.

6. Del Buono A, Chan O, Maffulli N. Achilles tendon: functional anatomy and novel emerging models of imaging classification. Int Orthop. 2013;37:715-721.

7. Webborn N, Morrissey D, Sarvananthan K, Chan O. Acute tear of the fascia cruris at the attachment to the Achilles tendon: a new diagnosis. Br J Sports Med. 2014.

8. Padulo J, Oliva F, Frizziero A, Maffulli N. Muscles, Ligaments and Tendons Journal. Basic principles and recommendations in clinical and field science research. MLTJ. 2013;4:250-252.

9. Beggs I, Bianchi S, Bueno A, et al. European Society of MusculoSkeletal Radiology: Musculoskletal Ultrasound Technical Guidelines: Ankle. 2013.

10. Fredberg U, Bolvig L, Andersen NT, Stengaard-Pedersen K. Ultrasonography in evaluation of Achilles and patella tendon thickness. Ultraschall Med. 2008;29:60-65.

11. Peers KHE, Brys PPM, Lysens RJJ. Correlation between power Doppler ultrasonography and clinical severity in Achilles tendinopathy. Int Orthop. 2003;27:180-183.

12. Ohberg L, Lorentzon R, Alfredson $\mathrm{H}$. Neovascularisation in Achilles tendons with painful tendinosis but not in normal tendons: an ultrasonographic investigation. Knee Surg Sports Traumatol Arthrosc. 2001;9:233-238.

13. Morton S, Parkes T, Chan O, et al. Achilles Intratendinous Tears - A New Pathology? Results of a 4 year case series. (Poster) Presented at BASEM October 2013.

14. Leung JL, Griffth JF. Sonography of chronic Achilles tendinopathy: A case-control study. J Clin Ultrasound. 2008 36:27-32.

15. Franklyn-Miller A, Falvey E, McCrory P. Fasciitis first before tendinopathy: does the anatomy hold the key? (Editorial) $\mathrm{Br} \mathrm{J}$ Sports Med. 2009;43:887-889.

16. Paavola M, Paakkala T, Kannus $P$, Jarvinen M. Ultrasonography in the differential diagnosis of Achilles tendon injuries and related disorders. A comparison between pre-operative ultrasonography and surgical findings. Acta Radiol. 1998;39:612-619. 